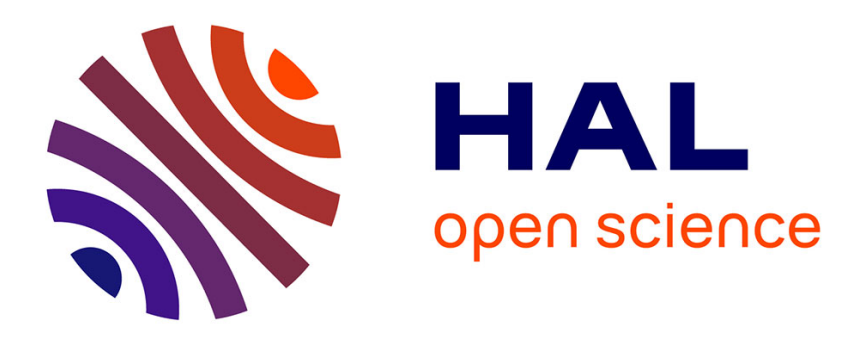

\title{
Peut-on habiter au vert quand le pétrole devient cher?
}

Xavier Desjardins

\section{To cite this version:}

Xavier Desjardins. Peut-on habiter au vert quand le pétrole devient cher?. Pour: enquêtes et témoignages, 2009, 199, pp.116-123. halshs-00472998

\section{HAL Id: halshs-00472998 \\ https://shs.hal.science/halshs-00472998}

Submitted on 13 Apr 2010

HAL is a multi-disciplinary open access archive for the deposit and dissemination of scientific research documents, whether they are published or not. The documents may come from teaching and research institutions in France or abroad, or from public or private research centers.
L'archive ouverte pluridisciplinaire HAL, est destinée au dépôt et à la diffusion de documents scientifiques de niveau recherche, publiés ou non, émanant des établissements d'enseignement et de recherche français ou étrangers, des laboratoires publics ou privés. 
Xavier Desjardins

\section{Peut-on habiter au vert quand le pétrole devient cher ?}

Articlé publié dans la revue POUR, n 199, février 2009, pp. 116-122

Si le cours du pétrole est reparti à la baisse, ce qui soulage quelques inquiétudes, tout indique qu'il va connaître une forte période de turbulence au cours de la prochaine décennie, pour des raisons géopolitiques (tensions dans les pays producteurs) et géologiques (épuisements progressifs des réserves). Comment les territoires ruraux et périurbains vont-ils être affectés par ces mutations ? Pour certains, les territoires à faible densité seraient condamnés par leur forte dépendance à une mobilité automobile énergivore. Toutefois, rien ne permet d'apporter une réponse assurée à cette question. Pour ce problème, comme pour tant d'autres, le pire n'est pas toujours sûr!

La question énergétique mettra-t-elle un terme au mouvement de dispersion des populations sur le territoire ? Depuis les années 1970, un très fort mouvement de desserrement urbain a conduit à un rapide développement des zones périurbaines et de leurs marches rurales, de plus en plus loin des centres urbains (Roux, Vanier, 2008). Aujourd'hui, ce sont les petites communes qui tirent le développement démographique du pays. Même le rural dit profond se repeuple parfois. Le développement de résidences secondaires n'y est pas étranger, parce que celles-ci deviennent parfois principales. Les données annuelles de l'Insee montrent ainsi qu'en 2006, l'ensemble des communes de moins de 1000 habitants a connu une croissance de plus de 105000 habitants, soit un rythme de croissance démographique annuelle supérieur à $1 \%$, tandis que l'ensemble des villes de plus de 10000 habitants ne connaît qu'un gain de population de 80000 habitants, soit un rythme annuel de croissance inférieur à 0,3\%.

Cette grande transformation territoriale s'est réalisée, de manière progressive, depuis une trentaine d'années. Elle fut en grande partie imprévue, si l'on se souvient que le fameux Paris et le désert français, rédigé en 1947 par Jean-François Gravier, a inspiré certaines politiques nationales d'aménagement du territoire. Les causes de cette mutation sont très diverses. Les ménages sont à la recherche de logements plus grands, que l'on trouve plus facilement dans les zones périurbaines et rurales : en une génération, entre 1972 et 2006, la surface de logement par habitant a progressé de 60 \% (Insee Première, 2008). La décentralisation et le 
transfert aux communes de compétences en matière d'urbanisme dans les années 1982-1983 ont permis à de nombreux élus de petites communes de développer une politique d'offre foncière très active afin de repeupler leurs villages en déclin et de redonner vigueur à la vie rurale pour sauver une école, faire vivre une salle des fêtes, etc. D'un autre côté, l'absence de politiques foncières importantes et anticipatrices et, donc, d'offres immobilières accessibles dans les agglomérations centrales, a conduit à un report de la demande de logement vers les zones moins denses.

Le talon d'Achille de cette transformation territoriale est sa dépendance à une énergie abondante et peu chère. En effet, ce développement a été permis par la baisse, sur la longue durée, des coûts du transport, notamment automobile. Son usage se révèle de moins en moins coûteux, avec une diminution du coût du kilomètre parcouru en automobile de $65 \%$ par rapport au SMIC horaire entre 1975 et 2005 (Beauvais, 2006). Cette transformation a également été facilitée par une amélioration constante des réseaux routiers. Les vitesses praticables sont de plus en plus élevées. La dernière enquête nationale de transport date de 1993. Elle a montré que si la durée des déplacements avait peu évolué depuis l'enquête précédente de 1982, avec en moyenne 55 minutes par jour et par personne de plus de six ans, le nombre de kilomètres parcourus s'était accru de plus de $30 \%$ en une décennie. Entre les deux dates, les ménages ont changé de mode de transport, en faveur des plus rapides, notamment de l'automobile. Aussi, de nombreux ménages peuvent-ils habiter dans une petite commune tout en ayant un emploi au cœur d'une agglomération distante d'une vingtaine de kilomètres. Cette facilitation de la mobilité transforme également les pratiques de loisir. Comme les déplacements sont moins coûteux et plus confortables, les départs en week-end ou pour de courts séjours sont de plus en plus nombreux, permettant des flux temporaires de population dans les espaces à faible densité.

À la fin des années 1990, le législateur, à travers notamment la loi Solidarité et renouvellement urbains, a souhaité lutter contre cette tendance à une urbanisation de plus en plus diffuse par une relance de la planification territoriale. De nombreux documents d'urbanisme, notamment les schémas de cohérence territoriale, ont affiché comme objectif de lutter «contre l'étalement urbain », sans toujours bien distinguer ce qui ressortissait d'un étalement de la tache urbaine (qui entraîne une réduction des espaces agricoles et naturels) de ce qui était un étalement fonctionnel (qui entraîne un accroissement des distances parcourues par les habitants pour l'accès aux différentes ressources du territoire). Si la planification a été 
vigoureusement relancée, les transformations du territoire observées ne sont pas du tout en adéquation avec les objectifs alors affichés au nom du développement durable : la ville compacte tant désirée semble fuir (Desjardins, 2008a). Mais ce que la planification appelait à faire, de manière un peu incantatoire, au nom du développement durable, ne va-t-il pas se réaliser de manière beaucoup plus certaine par la contrainte énergétique? En effet, de nombreuses études ont montré que plus les ménages habitent loin des centres urbains, plus leur budget consacré aux déplacements est élevé (Orfeuil, Polacchini, 1998). Aussi, en toute bonne logique, pourrait-on s'attendre à ce que, dans les moments de hausse du coût du carburant, on assiste à une rétractation périurbaine, ou au moins, à un déclin de l'attractivité résidentielle de ces territoires. De même, dans un contexte de contraintes budgétaires pour les déplacements, l'attractivité des activités de loisir pourrait décliner dans les territoires ruraux. Bref, la distance, qui était devenue une notion presque secondaire derrière celle du temps dans les pratiques territoriales des ménages et des entreprises, va-t-elle redevenir une variable fondamentale ? Différents éléments nous montrent que ce scénario n'est peut-être pas le plus probable.

Quelques données statistiques montrent une légère mutation des pratiques de mobilité. Deux enquêtes auprès des ménages sur les déplacements réalisés en 2006 montrent, pour les agglomérations de Lille et de Lyon, un retournement de tendance avec une mobilité quotidienne en automobile en diminution par rapport aux années 1990 (Quetelard, 2008). D'autres données concernant la mobilité sur longue distance, c'est-à-dire de plus de 100 kilomètres, montrent pour l'année 2006 un recul du nombre de kilomètres parcourus en automobile d'un peu plus de $2 \%$. Néanmoins, si ces chiffres marquent une certaine sensibilité des ménages au prix du carburant, il est trop tôt pour savoir si on assiste à un simple effet de conjoncture ou à un bouleversement structurel plus profond. Aux États-Unis, où la hausse des coûts du carburant a été beaucoup plus massive qu'en Europe entre 2003 et 2008 avec un quasi-triplement du prix de l'essence, on observe une diminution du nombre de kilomètres parcourus en automobile de près de $6 \%$ pendant la même période. Il y a donc une diminution passagère, mais en 2008 le nombre de kilomètres automobiles parcourus par habitant et par an est toujours supérieur de près de $40 \%$ à celui de 1983 (Desjardins, 2008b) ! On voit donc quelques signes de changements, mais, pour l'instant, il est encore trop tôt pour annoncer une transformation profonde du système territorial. 
Pourquoi l'impact de la hausse du coût de l'énergie sur le territoire paraît-il encore peu massif ? Tout d'abord, il existe de très fortes réserves de capacité pour améliorer l'efficacité énergétique des automobiles. Entre 1970 et 2005, si le nombre de véhicules-kilomètres parcourus a été multiplié par 2,5 en France, la consommation énergétique a été seulement multipliée par deux grâce à une amélioration des technologies. On peut supposer que les constructeurs vont fortement accélérer la mise au point de véhicules plus sobres dans un contexte de hausse durable des coûts de l'énergie (et de contraintes légales liées à la politique de lutte contre le réchauffement climatique et de diminution des émissions de gaz à effet de serre). Les ménages peuvent ainsi répondre à une hausse durable du coût du carburant, non par une diminution de leur kilométrage automobile, mais par le choix de véhicules plus sobres. De plus, rappelons que si le carburant est l'élément déterminant du coût psychologique de l'usage d'un véhicule, il ne constitue toujours qu'une part minoritaire du coût réel de son utilisation (souvent moins d'un quart). Ensuite, les ménages ont, au sein de leur budget, une capacité de reporter l'effet de la hausse du coût de l'énergie sur d'autres postes de consommation que la mobilité. Un résultat inattendu d'une enquête exploratoire que nous avons menée dans le périurbain francilien en 2008, est que la principale réponse apportée par les ménages à la hausse du coût du carburant est... de réaliser des travaux d'amélioration thermique de leur logement (Desjardins et alii 2008)! Enfin, les inquiétudes, liées aux difficultés avérées pour les ménages vivant dans les zones à faible densité à pouvoir accéder aux ressources territoriales (emploi, éducation, loisir, commerce, services, etc.) en raison d'une chute brutale de leur mobilité, sont fondées par rapport au système territorial actuel. Celui-ci est marqué par la polarisation - en diminution, mais néanmoins forte - des emplois dans les métropoles ainsi que par la concentration continue des services, publics comme commerciaux, dans les plus grandes villes. Dans un contexte de cherté durable de l'énergie, on peut imaginer le développement d'autres formes de commerce, une réouverture des services publics de proximité, le développement de circuits plus courts pour la distribution de produits alimentaires ou nécessaires au bâtiment, une revitalisation des bourgs et des villes moyennes, le développement du covoiturage, une polarisation du développement résidentiel sur les communes desservies par des lignes de transport collectif. Sur ces différents points, les tendances actuelles et les politiques menées vont pratiquement toutes dans le sens strictement inverse, mais la question énergétique pourrait amener à refaire avec autant de passion ce qu'on s'acharne à défaire... 
L'insuffisante connaissance du présent rend très délicate la prospective en matière territoriale. Si on dispose de quelques éléments sur les effets de la hausse du coût du carburant, et nous avons mentionné certains travaux sur ce point, l'ampleur des inconnues est forte. Les enquêtes sur les déplacements des ménages ne concernent, avec une périodicité faible, que les grandes agglomérations. La mobilité rurale et périurbaine (ainsi que la consommation énergétique associée) est mal connue. La dernière enquête nationale sur le transport date de 1993. Une nouvelle enquête est actuellement conduite, mais il faut en attendre les résultats.

Pour l'avenir, les scénarios doivent prendre en compte les évolutions technologiques et les mutations des valeurs; or celles-ci sont loin d'être facilement prévisibles. Les évolutions technologiques vont-elles permettre de se déplacer demain sans utiliser d'énergie fossile ? En matière de mobilité, beaucoup pensent pouvoir trouver dans l'électricité un substitut énergétique au pétrole. La production électrique émet peu de gaz à effet de serre en France, principalement en raison de l'importance du parc nucléaire. Les moteurs électriques sont aujourd'hui adaptés aux véhicules automobiles. Toutefois, ces véhicules électriques sont encore très coûteux en raison des équipements nécessaires, notamment pour les véhicules hybrides qui fonctionnent à l'essence et à l'électricité. De plus, la fabrication ne concerne encore que des séries limitées. Enfin, il faudrait implanter des points de recharge, d'autant plus nombreux que l'autonomie des moteurs est limitée. Bref, si cette voie est possible, elle n'est sérieusement envisageable que dans quelques décennies et, pour Pierre Merlin (Merlin, 2008), elle repose nécessairement sur un renforcement du parc nucléaire.

Une autre grande inconnue concerne l'évolution des valeurs des ménages. Si nous n'avons évoqué, depuis le début de l'article, que le problème de la sensibilité des ménages au coût de l'énergie, nous ne pouvons ignorer, qu'outre la question économique, la maîtrise des consommations d'énergie est aussi une question environnementale. Les pratiques vont-elles changer de manière forte en raison de l'importance des débats sur le réchauffement climatique ? Les enjeux de la réduction des émissions de gaz à effet de serre vont-ils entraîner une nouvelle appréhension des risques et redonner une vigueur socialement acceptée à l'industrie nucléaire? Les énergies renouvelables vont-elles pouvoir se développer de manière soutenue, et dans ce cas, avec quels impacts sur les paysages et le foncier? On pourrait multiplier ainsi les questions à l'infini, mais là n'est pas le but. L'important est de montrer que la question énergétique est fondamentalement une question politique, puisqu'elle renvoie aux risques (environnementaux, géopolitiques, économiques...) que nous sommes prêts à 
assumer selon le type de développement que nous souhaitons. Il est donc fondamental que ce débat ne reste pas cantonné à une sphère technique qui en explique peu les conséquences sociales et territoriales, ou que ce débat, très peu consensuel sur certains aspects comme le nucléaire, les énergies renouvelables ou les modes de consommation, ne soit pas étouffé par des propos lénifiants sur le développement durable.

Au final, les territoires ruraux et périurbains vont-ils être affectés par la question énergétique ? Bien sûr, et à court terme, on observe des changements dans les pratiques des ménages et des situations de grandes difficultés pour les ménages les plus pauvres dans ces zones où l'automobile est le mode de déplacement presque exclusif pour les distances supérieures au kilomètre. Le plus probable est qu'à moyen terme, la hausse des coûts de l'énergie entraîne, non un déclin généralisé des territoires peu denses, mais une polarisation du développement sur les bourgs et les communes desservis par des transports collectifs. Le moment où chaque commune d'un canton pouvait accueillir de manière presque égale une petite part de la croissance démographique est certainement terminé dans la mesure où polariser le développement dans les communes disposant déjà de services étoffés et d'un passage de lignes d'autobus paraît une sage prudence. L'intercommunalité pourra apporter une aide concrète à cette réorientation vers un développement moins diffus dans le territoire. Lors de l'élaboration des documents d'urbanisme, notamment des schémas de cohérence territoriale, il peut être intéressant, avant de choisir la localisation des zones à urbaniser, d'essayer d'évaluer le coût énergétique prévisible que devront supporter les nouveaux résidents afin de choisir les zones les moins énergivores à l'échelle d'une intercommunalité ${ }^{1}$. Pour le plus long terme, ce qui est sûr, c'est que la manière dont les citoyens se saisiront (ou non) de cette question aura des répercussions territoriales majeures.

\footnotetext{
${ }^{1}$ Des modèles très sophistiqués de calcul existent, mais on peut également partir de modèles très simples en prenant une famille-type de quatre personnes avec deux actifs et deux enfants : on prend par convention l'idée que les deux adultes doivent parcourir pour leur travail une distance égale à la distance moyenne parcourue par les résidents actuels, puis on ajoute les distances nécessaires pour l'école, les activités de loisir et les achats. On aboutit à un nombre de kilomètres en automobile et en transport collectif auxquels ce ménage-type est contraint, donc à un coût monétaire et à une quantité d'énergie. Ce modèle manque beaucoup de finesse dans la mesure où les ménages-types n'existent pas et où les pratiques ne sont jamais aussi simples. Néanmoins, il permet en quelques minutes de voir lors du débat sur les zones à ouvrir à l'urbanisation, si certaines se révèlent $a$ priori plus ou moins énergivores. Sans surprise, les zones proches des commerces et services et à proximité des arrêts de transports collectifs se révéleront alors plus intéressantes, mais remarquons que ce ne sont pas toujours celles qui sont choisies pour l'urbanisation future...
} 


\section{À lire}

Jean-Marie Beauvais, «Prix réel des carburants et transports collectifs urbains : évolution 1970-2005 », Transports Urbains n¹12 mars 2008, p. 3-8.

Xavier Desjardins, «Ville rêvée et ville réelle. Veut-on réellement lutter contre l'étalement urbain ? », Études foncières, janvier-février 2008a, p. 16-19.

Xavier Desjardins, «Les chiffres des effets de la hausse du prix des carburants sur la mobilité des Américains. Le prix de l'essence, révélateur de tendances lourdes? », Transports Urbains $\mathrm{n}^{\circ} 114$ décembre 2008b, p. 34-35.

Xavier Desjardins et al., Le développement périurbain au péril de la crise énergétique ? Note de recherche, CSTB, décembre 2008 (non publié).

Pierre Merlin, Énergie et environnement, La Documentation française, 2008.

Bernard Quételard, «Du nouveau dans le partage modal », Transports urbains $\mathrm{n}^{\circ} 112$, mars 2008, p. 9-12.

Jean-Pierre Orfeuil, Annarita Polacchini, «Les dépenses des ménages franciliens pour le logement et pour les déplacements », Recberche Transport Sécurité n 63, avril-juin 1999, p. 31-46.

Emmanuel Roux, Martin Vanier, La périurbanisation : problématiques et perspectives, La Documentation française, Diact, 2008, 87 p.

Bénédicte Castéran, Layla Ricroch, «Les logements en 2006 », Insee Première $n^{\circ} 1202$, 2008. 